\title{
Tool Wear Evaluation Based on Design Space Exploration Coupled with Explicit Cutting Simulations and Cutting Forces Excitation Signature
}

\author{
Andrei - Ionut BERARIU \\ Transilvania University of Brasov, Romania, andrei-ionut.berariu@unitbv.ro \\ Iulia - Maria PRODAN \\ Transilvania University of Brasov, Romania, iulia-maria.prodan@unitbv.ro \\ Sebastian - Stefan GOROBIEVSCHI \\ Transilvania University of Brasov, Romania, sebastian.gorobievschi@student.unitbv.ro \\ Tudor - Ion DEACONESCU \\ Transilvania University of Brasov, Romania, tdeacon@unitbv.ro
}

\begin{abstract}
Cutting operations are still one of the main methods used in the industry for surface generation in mass production. The dynamics of these processes are complex and having a good insight into the interdependencies of the nonlinear physical phenomena can be translated into better cutting performance. One of the main cutting tool geometrical parameter, usually associated with wear, is the cutting edge radius. Continuous direct evaluation of this parameter exhibits some important limitations offline, if this evaluation needs to be online the whole process becomes very complicated due to the measurement limitations that might appear. For online cases, the best approach is to determine the amplitude of this parameter indirectly using various side effects that can be correlated. One possible option is to monitor the vibrations generated by the resulting cutting forces.

In the latest years, the usage of explicit finite element methods (FEM) to simulate the cutting processes has grown exponentially with the continuous increase of computation efficiency. With the help of Computer-Aided Engineering (CAE in short) solutions and using the latest advances in design space exploration (DSE) solutions, it is possible to create models able to parametrically explore a design space (DS), having precise targets, and also get the important correlations for all the important, quantifiable, cutting parameters.

This paper presents an innovative method to create tool wear evaluation models focused mainly on the cutting edge radius indirect evaluation. The proposed method uses guided simulation loops able to generate a variety of dynamic signatures which are further post-processed to get a complex correlative model. The model can be applied in real cutting cases using the reciprocity property and can determine online the state of the cutting edge radius for further tool wear evaluation. The paper concludes with an analysis of the obtained model and the applicability of the data for the intended purpose.
\end{abstract}

\section{Keywords}

design space exploration (DSE), explicit cutting simulation (CAE), tool wear, cutting edge radius, cutting forces

\section{Introduction}

Some of the oldest surface-generation processes that are still used today are caught under one big category, machining. The fundamental characteristic of a machining process is the controlled removal of raw material from the workpiece using a cutting tool and it is graded by the material removal rate (MRR) parameter.

The wide variety of cutting processes (turning, boring, drilling, milling, broaching etc.), each possessing specific geometric, kinematic, tool etc. requirements, creates a very complicated environment for which unified control techniques can be applied. The present paper focuses only on one small (general) aspect that every cutting tool possesses, cutting edge radius $r$, strongly linked to the tool wear. In order to build the proof of concept, the present research is concentrated on the face turning operation of AISI 1045 steel.

There is a great interest from the academic community to understand how the flank wear evolves through the tool-life cycle, how to optimized growth rate and finally how to detect when it reaches a critical level from which it can compromise a workpiece. The main difficulty is the fact that this geometric aspect of the tool is practically in the most inaccessible place, where the cutting takes place, making it extraordinarily difficult to measure during the cutting. In their work, Patel V.D. et al [1], 
modeled the cutting process of finish turning of hardened AISI D2 steel taking into account also the flank wear. One of the main conclusions was that the cutting forces can be predicted with more accuracy when this parameter is taken into consideration with experimental validation that showed a prediction error between 2.24 and 6.4\%. Suyama D.I. et al [2] studied the influence that the vibrations can have on tool wear mechanism, very important in the context of the present work, for turning operations of hardened steels with intriguing results. The research concluded, among other things, that the induced vibrations are not influencing the tool life but rather it has a great impact on the wear mechanism. When the amplitudes of the vibrations were high, diffusion was the predominant mechanism and when the amplitude was low the abrasion was the main mechanism.

There is also another approach that is becoming more and more popular with the Industry 4.0 movement and that is the usage of artificial intelligence (AI). Serin G. et al [3] present a very detailed study for the state of the art for this new emerging technology focused on tool condition monitoring. A dedicated study for the application of AI in tool wear prediction for hard turning operations can be found in [4]. Thangarasu S.K. et al managed to predict the tool wear for EN8 steel using the cutting forces and the roughness of the obtained surface using an artificial neural network. The team also concluded that the tool wear was greatly influenced by feed $\mathrm{f}(61.63 \%)$ and slightly by the depth of cut doc and cutting speed V (all terms are explained and used later). There is also research focused on other, more complex, operations like milling. Wu T.Y. et al [5] managed to predict with enough accuracy the workpiece roughness using the machine vibrations and the cutting parameters.

The present paper proposes a novel method to create tool wear models for any cutting operations and although, for now, it is exposed only for the turning -face cutting operation of AISI 1045 steel within some limited cutting parameters, the method should be applicable, with the current adjustments, for any machining operation. If the machining operation can be modeled using computer-aided engineering (CAE) solutions then, theoretically, it is possible to enclose the variation space of all the cutting parameters in an design space (DE) that can be picked up by an design space exploration (DSE) product and explored determining also all the interdependencies/correlations that the input/output parameters have, thus connecting some easily measurable phenomena with the tool wear state.

\section{Design Space Exploration and Synthetic Sample Generation}

The proposed method uses synthetic cutting data generated by the synergy created between CAE and DSE products, described herein, that are not perfectly compatible for the intended purpose.

The universality of the solutions, especially the DSE ones, requires some very specific tweaks to form the complete ecosystem where the simulation loop can function. In the following, all the critical aspects required to define a model of this kind will be exposed (Figure 1).

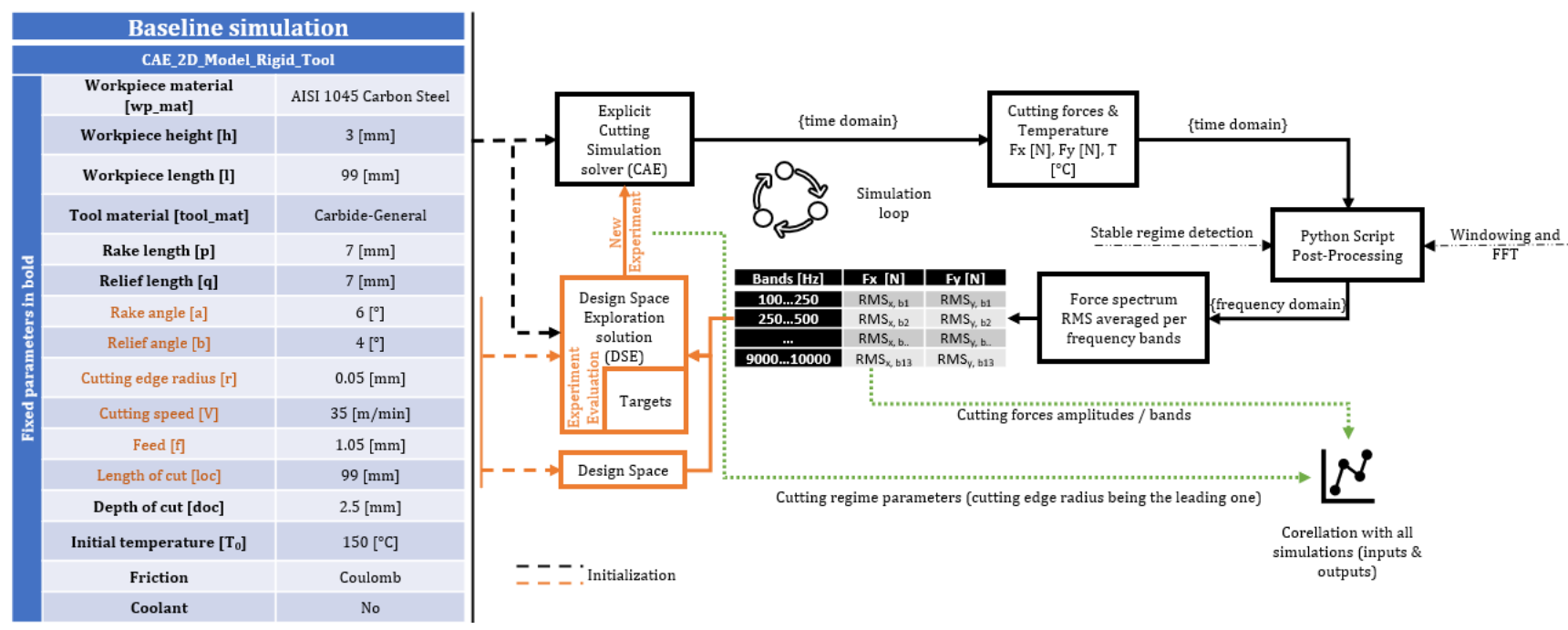

Fig. 1. DSE - CAE cutting simulation loop with cutting edge radius being the main tracking parameter 
The core of any DSE solution lays in the method that explores, evaluates trends and builds new simulation cases, all based on the DS dimensions and the targets. In this case, the most common parameters to increase the overall MRR rate were chosen as targets: increase the $V$ and $f$ and reduce the RMS values for all frequencies bands (more details in the following chapters).

\subsection{Turning, main kinematic and dynamic characteristics}

The main kinematic parameters in case of face turning are $N$ [rpm] - spindle speed, $f[\mathrm{~mm} / \mathrm{rev}]$ - feed, and doc [mm] - deep of cut. To compute the most important cutting parameter, $V$ - cutting speed $[\mathrm{m} / \mathrm{min}]$, the $D[\mathrm{~mm}]$ - workpiece diameter is needed such that (Figure 2 - left side):

$$
V[\mathrm{~m} / \mathrm{min}]=\frac{D[\mathrm{~mm}] \cdot \pi \cdot N[\mathrm{rpm}]}{1000}
$$

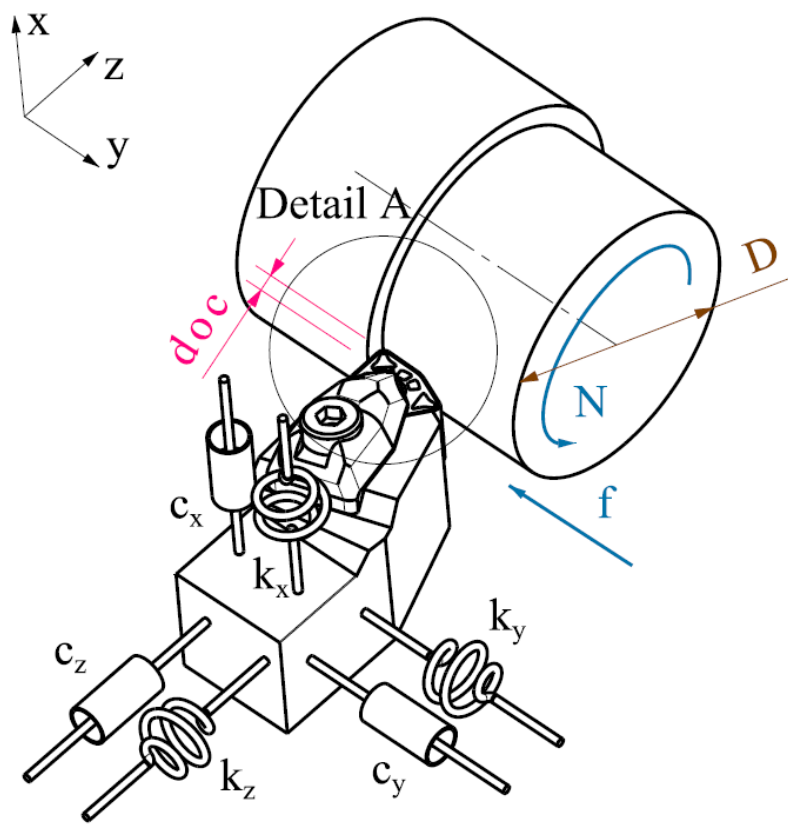

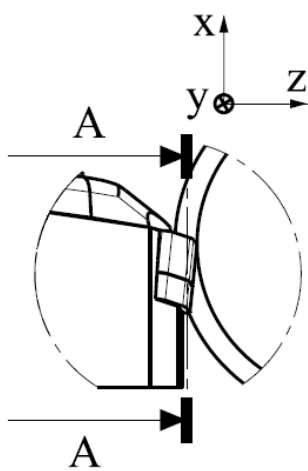

A

Detail A

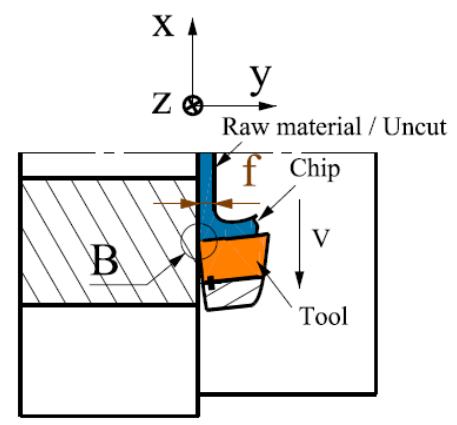

Section A - A

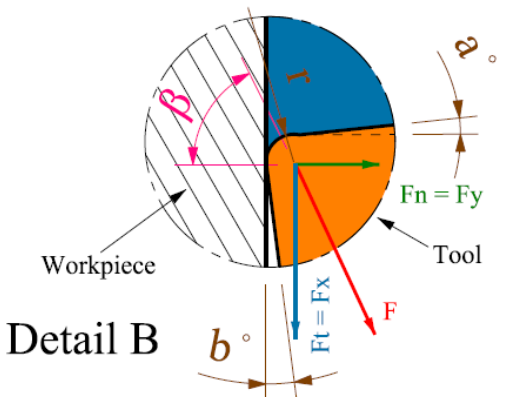

Fig. 2. The main kinematic and geometric parameters for face turning operation, the transition from oblique cutting (left) to orthogonal cutting (right)

The chip section (Figure 2 right), directly linked with the cutting behavior of the operation, is geometrically dependent on $f$ - feed and $d o c$ - deep of cut. Together with $a\left[^{\circ}\right]$ - rake angle, $b\left[{ }^{\circ}\right]-$ relief angle, $r[\mu \mathrm{m}]$ - cutting edge radius and the physical properties of the tool-workpiece interface, the cutting process can be simplified, from a 3D mechanical problem to a 2D problem, using the orthogonal approach.

This simplification reduces the cutting force vector by dropping the $\mathrm{Z}$ direction and keeping only the main two cutting forces, the normal force $F n=F x$ and the tangential force $F t=F y$, for the study.

The literature already provides some insights into the effect that the cutting edge radius $r$ might have on the overall cutting dynamics. This parameter can have a great impact on the effective rake angle $a$ especially when the value of it approaches the same order of magnitude as feed $f$. When this happens, it is possible to see an effect of sing flipping for the rake angle $a$ which has a great impact on $F x$ and $F y$ distribution and characteristics (Figure 3). Furthermore, the negative rake angle serves to impose compressive stresses on the workpiece surface leading to negative effects not just on the tool wear process but also on the workpiece quality [6]. 

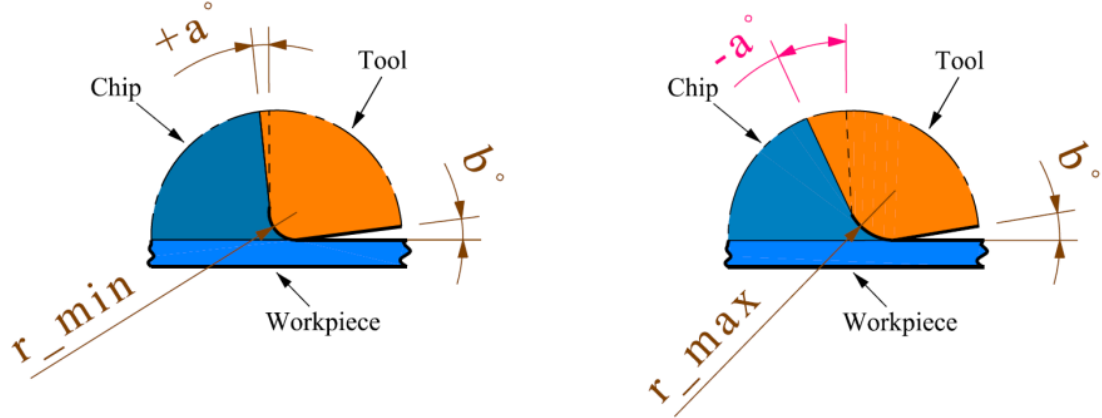

Fig. 3. Effect of cutting edge radius on effective rake angle. When the uncut chip thickness is larger compared with the edge radius, the effective rake angle flips signs

\subsection{Explicit simulation for cutting, orthogonal cutting}

In terms of optimization, oblique problem to orthogonal problem conversion, it was demonstrated in [7] that, if applied correctly, this approach can reduce the computation effort by $90 \%$ thus generating a lot more samples per time/resources unit.
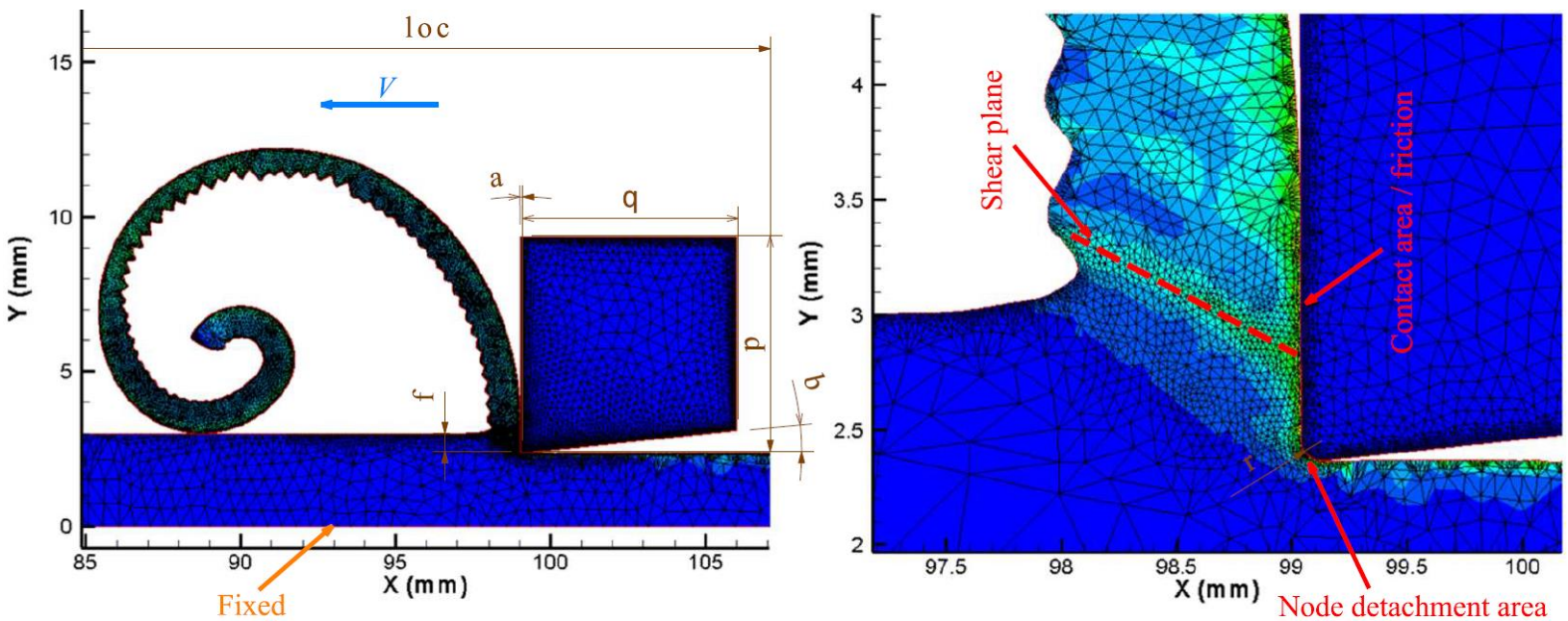

Fig. 4. Orthogonal cutting simulation, critical dynamic aspects

The usage of CAE simulation requires knowledge of the following parameters: $V[\mathrm{~m} / \mathrm{min}], a\left[^{\circ}\right], \mathrm{b}\left[{ }^{\circ}\right]$, $r[\mu \mathrm{m}], \mathrm{f}[\mathrm{mm}], d o c[\mathrm{~mm}]$ and the $w p_{-} m a t$ - workpiece material (Figure 1), $f$ no longer expressed in $[\mathrm{mm} / \mathrm{rev}]$ but in $[\mathrm{mm}]$ due to the conversion. The cutting tool is considered rigid and the Coulomb friction coefficient is used, thus the tool_mat - tool material is defined too as Carbine - General.

Commonly, the meshing process for CAE - FEM (Finite Element Method) implies the usage of triangles and/or quadrangles elements for the discretization of the physical domains (Figure 4). Besides the complexity of the material properties definition, usually dependent on various other phenomena, this meshing process needs to be updated continuously with respect to the mechanical state of the chip. This is a particularly difficult problem to control and the literature offers multiple options to control this, the most common one being the Arbitrary Lagrangian Formulation (ALE), more details in [8].

The main objective for the CAE component of the DSE-CAE loop is to catch in the Fx and Fy dynamic signature for the design space (DS) domain with respect to $r$. In order to facilitate this process, the mesh density of the node detachment area needs to be finer but also the shear plane needs to be controlled to avoid having noise in the signal caused by the effect of share plan detachment.

\subsection{Post-processing the cutting results and coupling the results to the DSE - CAE simulation loop}

The following parameters are considered for the CAE component and not for the DSE-loop component since they are constants: wp_mat - workpiece material, $h[\mathrm{~mm}]$ - workpiece height, $l[\mathrm{~mm}]$ - workpiece length, tool_mat - tool material, $p[\mathrm{~mm}]$ - rake length, $q[\mathrm{~mm}]$ - relief length, 
doc $[\mathrm{mm}]$ - deep of cut, $T_{0}\left[{ }^{\circ} \mathrm{C}\right]$ - initial temperature, friction and the usage of coolant (Figure 1 - left, bold). The design space for the DSE loop will be formed by: $a\left[{ }^{\circ}\right]$ - rake angle, $b\left[^{\circ}\right]-$ relief angle, $r$ [mm] - cutting edge radius, $V[\mathrm{~m} / \mathrm{min}]$ - cutting speed, $f[\mathrm{~mm}]$ - feed and loc $[\mathrm{mm}]$ - length of cut (Figure 1 - left, orange).

In order to get the dynamic signature of each simulation, the time domain signal $(F x$ and $F y)$ is transformed into a frequency domain signal using Discrete Fourier Transformation (DFT). Furthermore, to reduce the dimensions of the DS, the frequencies spectrum are RMS averaged per frequency bands in order to have an overall, banded, energy characteristic.

The entire process of converting the time domain data obtained from the CAE simulation into a frequency domain spectrum ready to be injected back to the CAE-DSE loop was specifically designed by the authors of this paper using Python ${ }^{\mathrm{TM}}$.

The nature of the simulation, explicit cutting with transient and stable regimes, exhibits the first impediment that needed to be handled in the post-processing component. For this detection, it was decided that the temperature variation can be used as a tracking value due to the nature of the deformation process. In the new Python program, a mechanism able to detect the first time step where the temperature stays constant within a threshold (in this case +/-5\%) and then selects the data based on that time step was implemented to solve this issue (Figure 5).
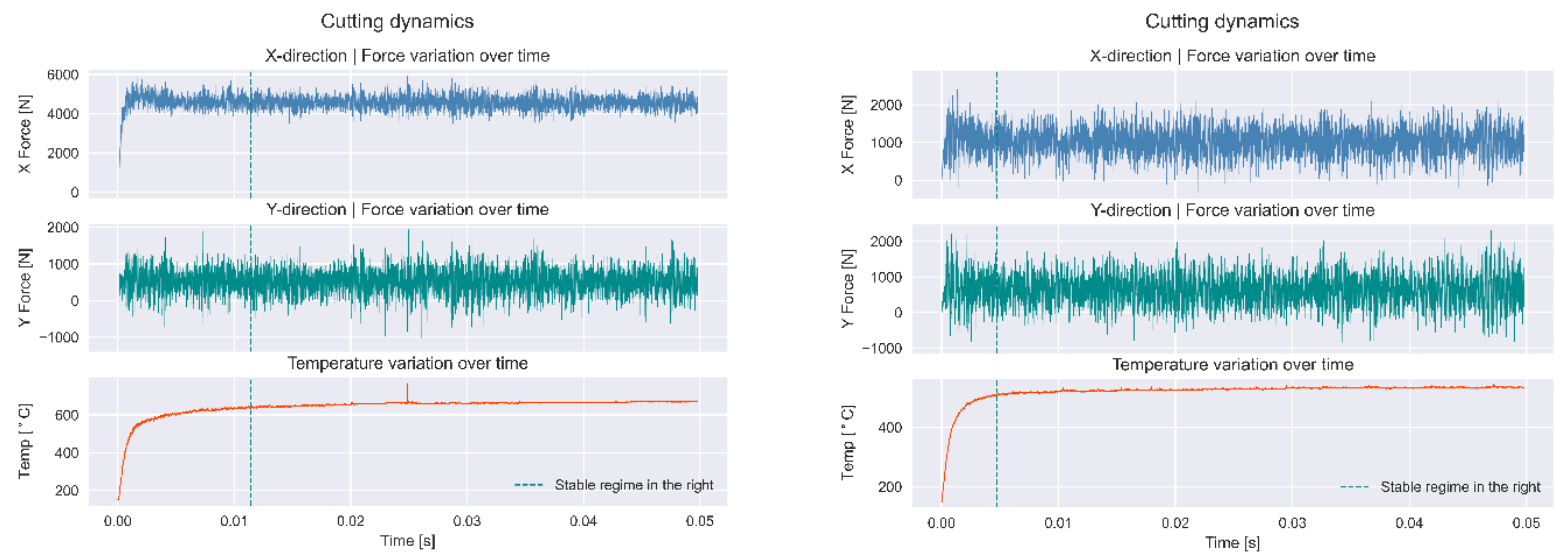

Fig. 5. The first stage of Python script post-processing, reading the force data and stable regime detection for: $V=34.117 \mathrm{~m} / \mathrm{min}, f=0.917 \mathrm{~mm} / \mathrm{rev}, r=20 \mu \mathrm{m}$ (left), and $V=23.473 \mathrm{~m} / \mathrm{min}$, $f=0.1 \mathrm{~mm} / \mathrm{rev}, r=90 \mu \mathrm{m}$ (right)

After the stable faze detection, the DFT conversion is applied using the commonly used Hanning windowing. The final step was to split the de obtained frequency spectrum into bands of interest and applying an RMS average for each band to obtain an overall energy characteristic e.g. $R M S_{x} b_{1}[\mathrm{~N}]-\mathrm{RMS}$ value for frequency band 1 in the $\mathrm{x}$-direction (Figure 1 ). The frequency bands were defined as following: $b_{1}=0.1 \ldots 0.25[\mathrm{kHz}], b_{2}=0.25 \ldots 0.5[\mathrm{kHz}], b_{3}=0.5 \ldots 0.75[\mathrm{kHz}], b_{4}=0.75 \ldots 1[\mathrm{kHz}], b_{5}=1 \ldots 2[\mathrm{kHz}]$, $b_{6}=2 \ldots 3[\mathrm{kHz}], b_{7}=3 \ldots 4[\mathrm{kHz}], b_{8}=4 \ldots 5[\mathrm{kHz}], b_{9}=5 \ldots 6[\mathrm{kHz}], b_{10}=6 \ldots 7[\mathrm{kHz}], b_{11}=7 \ldots 8[\mathrm{kHz}]$, $b_{12}=8 \ldots 9[\mathrm{kHz}], b_{13}=9 \ldots 10[\mathrm{kHz}]$. This can be used to evaluate the dynamic behavior of the process (Figure 6), how it might impact the overall machine tool (MT) stiffness characteristic and also give an insight into the $r$ condition (first used to define the detection model and then for online monitoring).

\section{Results and discussion}

Using the described method, it was possible to generate 25 cutting samples for the proposed machining operation within three weeks of computation effort. The computations were done on a machine having the following characteristics: CPU Intel i7 $2.7 \mathrm{GHz}$, RAM $32 \mathrm{~Gb}$, SSD $512 \mathrm{~Gb}$.

Based on the obtained data, the correlation between the two cutting forces signatures and the state of the cutting edge radius $r$ can be extracted (Figure 7 and Figure 8). Analyzing the first plot, $F x$ correlation with $r$, some critical aspects about how the evolution of the cutting edge radius can be 
monitored becomes quantifiable. The low-frequency bands are the most sensitive to the evolution of $r$ having a distinguishable inverse proportionality characteristic (between band 1 and 9). Similarly, analyzing the second plot, Fy correlation with $r$, the situation is more complex. In this case, both inverse and direct proportionalities are coexisting in the banded spectrum, exposing an even more reliable coupling since the uniqueness of the signature will have a higher probability.
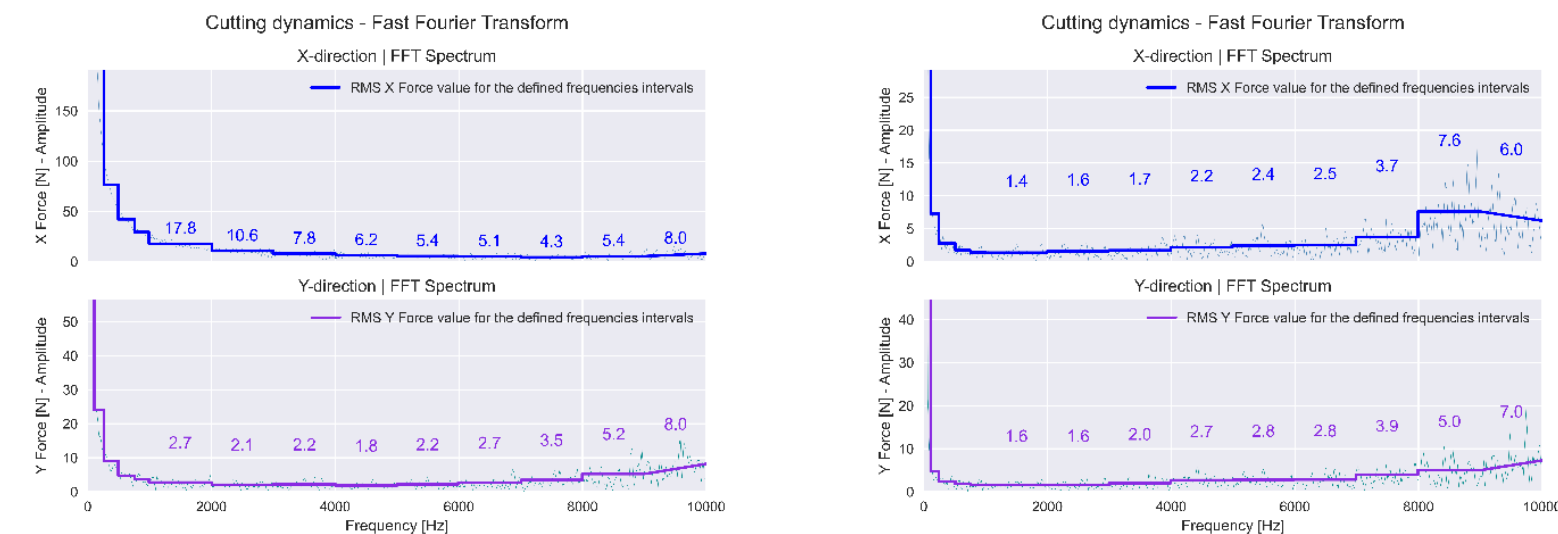

$V=34.117 \mathrm{~m} / \mathrm{min}, f=0.917 \mathrm{~mm} / \mathrm{rev}, r=20 \mu \mathrm{m}$

$V=23.473 \mathrm{~m} / \mathrm{min}, f=0.1 \mathrm{~mm} / \mathrm{rev}, r=90 \mu \mathrm{m}$

Fig. 6. Cutting forces spectrum results - RMS averaged for each frequency band

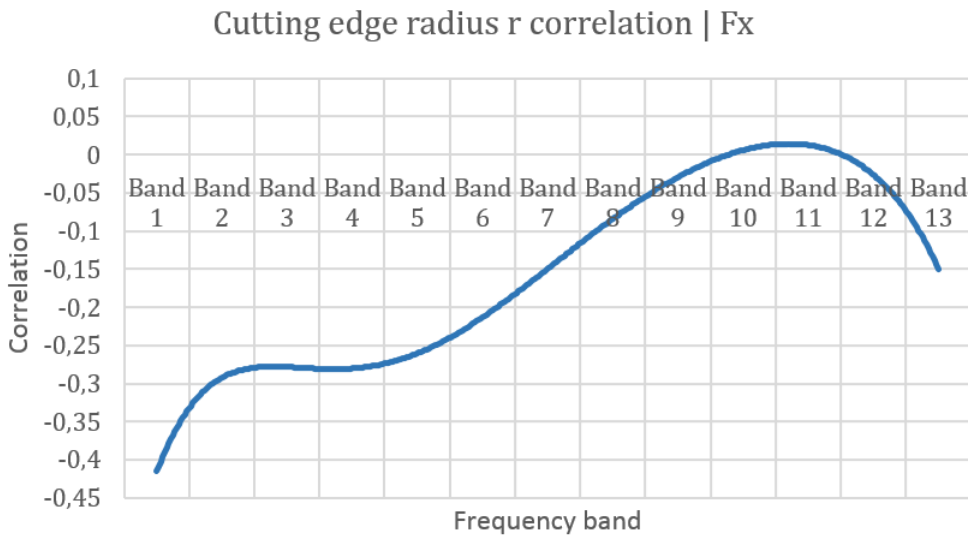

Fig. 7. Correlation plot, cutting dynamics characteristics of $F X$ vs cutting edge radius

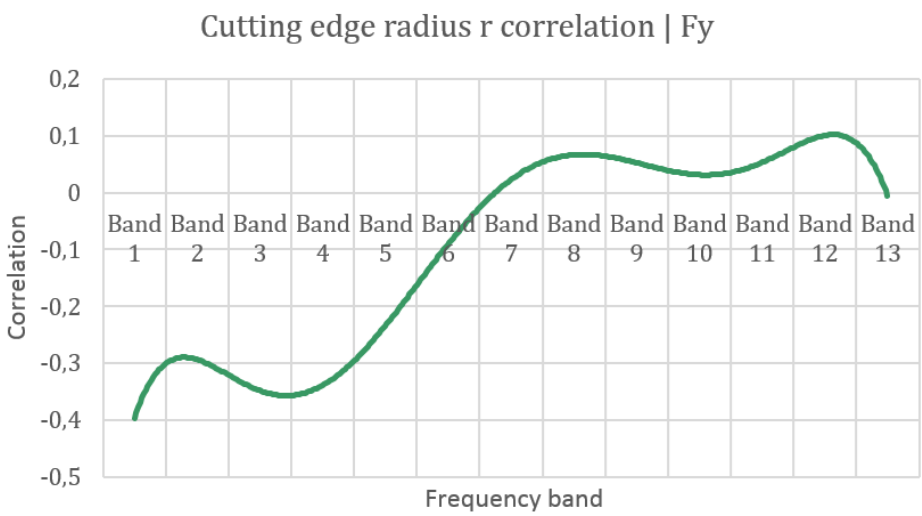

Fig. 8. Correlation plot, cutting dynamics characteristics of Fy vs cutting edge radius

The design space limits for the obtained data were set as follows: $a=-8 \ldots+8\left[^{\circ}\right]-$ rake angle, $b=0 \ldots 8\left[^{\circ}\right]-$ relief angle, $r=20 \ldots 100[\mu \mathrm{m}]-$ cutting edge radius [12], $V=1 \ldots 120[\mathrm{~m} / \mathrm{min}]-$ cutting speed, $f=0.1 \ldots 2[\mathrm{~mm}]-$ feed. 
Based on the correlation plots the conclusion is that the proposed method has the potential to expose very interesting and intertwined connections between the cutting force signatures and the state of the cutting edge radius $r$. It is also a scalable solution that can be adapted to any machining operation with the correct adjustments with one possible bottleneck in the form of CAE simulation capabilities.

\section{Conclusions}

The present paper is answering to the increasing demand for machining condition monitoring solutions for the critical aspects of cutting processes. The indirect monitoring of cutting edge radius is addressed and a new method that uses CAE and DSE solution is proposed. The objective is to define models able to analyze dynamic cutting signatures then correlate them with the state of the cutting edge radius for further processing.

The main component of this new method is the usage of Design Space Exploration (DSE) solutions connected to a Computer-Aided Engineering (CAE) solution. If the cutting problem can be modeled using quantifiable inputs and outputs correlated with a general scope, in this case reducing the vibrations, the DSE approach allows the construction of guided simulation loops able to generate dynamic signatures that are further correlated with the cutting edge radius behavior.

Besides the overall concept of coupling the two solutions (CAE and DSE), the authors are also presenting specific solutions developed in order to connect all DSE-loop components correctly. A new Python script was developed to execute critical operations like stable cutting regime detection, data selection, filtering, time-to frequency-domain transformation and exporting the results for the next simulation loop.

The obtained results are in good agreement with the proposed objectives and expose a possible new field to be investigated by other researchers that are working for similar models. Although no emphasis has been placed on numerical validation in this paper, the proof of concept has been made and the results obtained have suggested that this method is scalable and flexible enough to be used as a general tool.

\section{References}

1. Patel V.D., Gandhi A.H. (2019): Modeling of cutting forces considering progressive flank wear in finish turning of hardened AISI D2 steel with CBN tool. The International Journal of Advanced Manufacturing Technology, ISSN 0268-3768, Vol. 104, pp. 503-516, https://doi.org/10.1007/s00170-019-03953-2

2. Suyama, D.I., Diniz, A.E. (2020): Influence of tool vibrations on tool wear mechanisms in internal turning of hardened steel. Journal of the Brazilian Society of Mechanical Sciences and Engineering, ISSN 1678-5878, Vol. 42, is. 7, article number: 370, pp. 1-17, https://doi.org/10.1007/s40430-020-02452-w

3. Serin G., Sener B., Ozbayoglu A.M., Unver H.O. (2020): Review of tool condition monitoring in machining and opportunities for deep learning. The International Journal of Advanced Manufacturing Technology, ISSN 02683768, Vol. 109, is. 2, pp. 953-974, https://doi.org/10.1007/s00170-020-05449-w

4. Thangarasu S.K., Shankar S., Monhanraj T., Devendran K. (2020): Tool wear prediction in hard turning of EN8 steel using cutting force and surface roughness with artificial neural network. Proceedings of the Institution of Mechanical Engineers, Part C: Journal of Mechanical Engineering Science, ISSN 2041-2983, Vol. 234, is. 1, pp. 329-342, https://doi.org/10.1177\%2F0954406219873932

5. Wu T.Y., Lei K.W. (2019): Prediction of surface roughness in milling process using vibration signal analysis and artificial neural network. The International Journal of Advanced Manufacturing Technology, ISSN 0268-3768, Vol. 102, pp. 305-314, https://doi.org/10.1007/s00170-019-03317-w

6. Schmitz T.L., Smith K.S. (2019): Machining dynamics. Frequency Response to Improve Productivity. Springer International Publishing, ISBN 978-3-319-93706-9, DOI: 10.1007/978-3-319-93707-6, pp. 353-354, https://doi.org/10.1007/978-3-319-93707-6

7. Berariu A.I., Prodan I.M., Nita C.I., Deaconescu T. (2020): ANN Samples Generation Using 2D Dynamic FEM for Predicting Machining Vibrations. Acoustics and Vibration of Mechanical Structures - AVMS 2019, https://doi.org/10.1007/978-3-030-54136-1 39 (in press)

8. Adetoro O.B., Wen P.H. (2010): Prediction of mechanistic cutting force coefficients using ALE formulation. The International Journal of Advanced Manufacturing Technology, ISSN 0268-3768, Vol. 46, iss. 1-4, pp. 79-90, https://doi.org/10.1007/s00170-009-2079-7 